OPEN ACCESS

Edited by:

Wataru Aoi

Kyoto Prefectural University, Japan

Reviewed by:

Adeel Safdar,

Humber College, Canada

Jonathan Bogan,

Yale University, United States

Cameron Mitchell,

University of Auckland, New Zealand

*Correspondence:

Joris Hoeks

j.hoeks@maastrichtuniversity.n

Specialty section:

This article was submitted to Striated Muscle Physiology,

a section of the journal

Frontiers in Physiology

Received: 08 June 2017

Accepted: 04 September 2017

Published: 21 September 2017

Citation:

Dahlmans D, Houzelle A

Jörgensen JA, Phielix E, Lindeboom L,

Hesselink MKC, Schrauwen P and

Hoeks J (2017) Evaluation of Muscle

microRNA Expression in Relation to Human Peripheral Insulin Sensitivity: A

Cross-Sectional Study in Metabolically

Distinct Subject Groups.

Front. Physiol. 8:711.

doi: 10.3389/fphys.2017.00711

\section{Evaluation of Muscle microRNA Expression in Relation to Human Peripheral Insulin Sensitivity: A Cross-Sectional Study in Metabolically Distinct Subject Groups}

Dennis Dahlmans ${ }^{1}$, Alexandre Houzelle ${ }^{1}$, Johanna A. Jörgensen ${ }^{1}$, Esther Phielix ${ }^{1}$, Lucas Lindeboom ${ }^{1,2}$, Matthijs K. C. Hesselink ${ }^{1}$, Patrick Schrauwen ${ }^{1}$ and Joris Hoeks ${ }^{1 *}$

${ }^{1}$ Departments of Human Biology and Human Movement Sciences, Maastricht University Medical Center, Maastricht, Netherlands, ${ }^{2}$ Departments of Radiology, NUTRIM School for Nutrition and Translational Research in Metabolism, Maastricht University Medical Center, Maastricht, Netherlands

In recent years, several microRNAs (miRNAs)-post-transcriptional regulators of gene expression-have been linked to the regulation of peripheral insulin sensitivity. Many of these studies, however, have been conducted in cell or animal models and the few human studies available lack adequate measurements of peripheral insulin sensitivity. In the present study, we examined the expression of 25 miRNAs, putatively involved in (peripheral) insulin sensitivity, in skeletal muscle biopsies from extensively phenotyped human individuals, widely ranging in insulin sensitivity. To identify miRNAs expressed in skeletal muscle and associated with insulin sensitivity and type 2 diabetes, a comprehensive PubMed-based literature search was performed. Subsequently, the expression of selected miRNAs was determined by RT-qPCR using predesigned 384-well Pick-\&-Mix miRNA PCR Panel plates in muscle biopsies from type 2 diabetes patients, non-diabetic obese/overweight individuals, lean sedentary individuals and endurance-trained athletes. In all subjects, peripheral insulin sensitivity was measured by hyperinsulinemic-euglycemic clamp. The literature search resulted in 25 candidate miRNAs, 6 of which were differentially expressed in human type 2 diabetes compared to non-diabetic obese/overweight individuals. In turn, four of these miRNAs, i.e., miRNA27a-3p $(r=-0.45, p=0.0012)$, miRNA-29a-3p $(r=-0.40, p=0.0052)$, miRNA-29b-3p $(r=-0.70, p<0.0001)$ and miRNA-29c-3p $(r=-0.50, p=0.0004)$ demonstrated strong negative correlations with peripheral insulin sensitivity across all four subject groups. We identified miR-27a-3p and all members of the miRNA-29 family as potential regulatory players in insulin sensitivity in humans. These miRNA's may represent interesting novel targets for maintaining or improving insulin sensitivity.

Keywords: Type 2 diabetes, insulin sensitivity, microRNA, skeletal muscle, obesity 


\section{INTRODUCTION}

Type 2 diabetes mellitus (T2DM) is a chronic, progressive disease and diminished insulin sensitivity in peripheral tissues is an important phenomenon in its pathogenesis. Since skeletal muscle comprises $40-60 \%$ of total body weight and accounts for $80-90 \%$ of postprandial insulin-mediated glucose uptake (DeFronzo et al., 1985; Tseng et al., 2010), muscle tissue is of high metabolic significance. Therefore, understanding the factors that may contribute to muscle insulin resistance is important in order to find new opportunities to blunt the development of insulin resistance at an early stage. In this context, recent primarily preclinical studies, have linked microRNAs (miRNAs) to insulin sensitivity (Mohamed et al., 2014; Bork-Jensen et al., 2015; Dahlmans et al., 2016). miRNAs are noncoding RNA molecules of 18-24 nucleotides in length that post transcriptionally regulate many cellular processes by base pairing with conventional messenger RNAs (Bartel, 2004, 2009). Furthermore, most miRNAs are predicted to target a range of messenger RNAs, and can in turn be regulated by other miRNAs, underscoring the complexity of the regulation of protein expression by miRNAs. As such, miRNAs add a post-transcriptional and pre-translational layer to the complex regulation of adaptive responses in protein content. Thus, miRNAs play an important role over a wide range of cellular functions, including the regulation of metabolism (Krutzfeldt and Stoffel, 2006).

Indeed, several miRNAs have been linked to insulin resistance in both in vitro and in vivo models for insulin resistance. For example, miRNA-135a was elevated in skeletal muscle tissue of hyperglycemic $\mathrm{db} / \mathrm{db}$ mice whereas in vivo silencing of miRNA135a reduced hyperglycemia and improved glucose tolerance in $\mathrm{db} / \mathrm{db}$ mice, probably via inhibitory effects on IRS2 (Agarwal et al., 2013). In addition, miRNA-24 and miRNA- 126 were found to be significantly upregulated in skeletal muscle of insulin resistant Goto-Kakizaki rats as compared to normoglycemic Wistar rats (Huang et al., 2009). Furthermore, miRNA-194, determined by miRNA microarray analysis, was found to be reduced in T2DM patients as well as in insulin resistant rats (Latouche et al., 2016). Knockdown of miRNA-194 in L6 muscle cells improved insulin sensitivity, an effect that coincided with improvements in mitochondrial function (Latouche et al., 2016). Along similar lines, miRNA-149, shown to positively regulate $\mathrm{NAD}+$ and SIRT1 protein levels via direct interaction with poly (ADP-ribosyl) transferase-like 2 protein (PARP2), was shown to be decreased in high fat diet-induced insulin resistant mouse skeletal muscle (Mohamed et al., 2014). Finally, a study in C2C12 myotubes revealed that miRNA-106b expression was increased upon palmitic acid-induced insulin resistance while silencing of miRNA-106b improved mitochondrial health and promoted insulin sensitivity (Zhang et al., 2015). Besides these examples numerous other findings in primarily cell- and animal models link miRNAs to insulin sensitivity. In the present study, we thus performed a comprehensive literature search using the PubMed database, to select miRNAs expressed in skeletal muscle and associated with insulin resistance and T2DM.

Next, to establish human relevance of these candidate miRNAs, we aimed to examine if these miRNAs, putatively involved in insulin sensitivity, are differentially expressed skeletal muscle biopsies of thoroughly phenotyped, metabolically distinct subject groups that display a large range in peripheral insulin sensitivity and if their expression pattern could be linked to metabolic aberrations.

\section{METHODS}

\section{Subjects}

Samples were collected from 4 previously conducted studies; all performed at the department of Human Biology and Human Movement Sciences at Maastricht University (Phielix et al., 2010, 2012; van de Weijer et al., 2014; Vosselman et al., 2015). The institutional medical ethics committee approved the aforementioned studies [clinical trial reg. no. NCT00943059 (van de Weijer et al., 2014), NCT01298375 (Vosselman et al., 2015) and NTR2002 (Phielix et al., 2010, 2012)], and all participants gave their informed written consent in accordance with the Declaration of Helsinki. Four subject groups were included in the present study: (1) overweight/obese T2DM patients $(n=12)$; (2) non-diabetic obese/overweight individuals $(n=12)$; (3) young, lean sedentary individuals $(n=12)$ and (4) young, endurancetrained athletes $(n=12)$. Subjects were selected on the availability of muscle tissue and the presence of data for peripheral insulin sensitivity and in vivo mitochondrial function (i.e., PCr recovery rate and $\left.\mathrm{VO}_{2} \max \right)$ as main criteria. Subsequently, subjects were selected in order for the groups to match for age and BMI (i.e., lean sedentary subjects vs. endurance-trained athletes and overweight/obese, non-diabetic subjects vs. type 2 diabetic patients). All subjects were male, non-smoking and weight stable for at least 6 months. All T2DM patients were diagnosed with T2DM for at least 1 year, used metformin alone or in combination with sulfonylureas and were allowed to use statins as lipidlowering drugs. Patients were instructed to discontinue glucose lowering medication at least the week before the actual tests and biopsies, all other medications were continued during the studies. Finally, type 2 diabetic patients were allowed to use antihypertensive medication, such as ACE inhibitors and ATII antagonists. The non-diabetic overweight/obese individuals, did not use any medication, and were excluded if they suffered from uncontrolled hypertension, cardiovascular disease, liver dysfunction or if they had first degree relatives with T2DM. Fasting blood glucose along with oral glucose tolerance tests were performed to ensure the absence of non-diagnosed T2DM in the obese individuals. Lean, sedentary subjects were included if they participated in no more than $1 \mathrm{~h}$ of exercise per week for the last 2 years and if their $\mathrm{VO}_{2}$ max was lower than $45 \mathrm{ml} / \mathrm{min} / \mathrm{kg}$. Endurance-trained athletes were included if they participated in endurance training at least 3 times per week for the last 2 years and if their $\mathrm{VO}_{2}$ max was above $55 \mathrm{ml} / \mathrm{min} / \mathrm{kg}$. Depending on the study, body composition was either determined via hydrostatic weighing according to the method of Siri (1956), or via Dualenergy X-ray absorptiometry (DXA).

\section{Peripheral Insulin Sensitivity}

Insulin sensitivity was assessed using either a two-step or a one-step hyperinsulinemic-euglycemic clamp. In total, 8 
T2DM patients, 6 obese/overweight individuals, 7 lean sedentary individuals and 10 endurance-trained athletes were assessed using a two-step hyperinsulinemic-euglycemic clamp. Insulin sensitivity was assessed using a one-step clamp in 4 T2DM patients, 6 obese/overweight individuals, five lean sedentary individuals and two endurance-trained athletes.

Patients refrained from physical exercise 3 days before the glucose clamp. On the evening before the clamp, patients were provided with a standardized meal. All hyperinsulinemiceuglycemic clamps were performed after an overnight fast, the clamps were conducted according to the method of DeFronzo et al. (1979). In short, for the one-step clamp, subjects received a continuous insulin infusion of $40 \mathrm{mU} / \mathrm{m}^{2} / \mathrm{min}$, and a parallel glucose infusion (20\% Glucose) was gradually increased until a steady state in blood glucose was achieved. For the twostep clamp, participants received, a low-insulin infusion of 10 $\mathrm{mU} / \mathrm{m}^{2} / \mathrm{min}$ for $3 \mathrm{~h}$ until a steady state in blood glucose was reached. Thereafter, a high-insulin infusion of $40 \mathrm{mU} / \mathrm{m}^{2} / \mathrm{min}$ was performed for the duration $2.5 \mathrm{~h}$, after which steady state was reached. In the present study, we only present the glucose infusion rate (GIR) upon $40 \mathrm{mU} / \mathrm{m}^{2} / \mathrm{min}$ of insulin, corrected for both total body weight and lean body mass, since this is a measure for peripheral (i.e., skeletal muscle) insulin sensitivity. In our study, the expression of selected miRNAs is measured in skeletal muscle. Therefore, it is most relevant to relate the outcome of this analysis to the insulin sensitivity of that tissue.

\section{Mitochondrial Oxidative Capacity}

A routine incremental bicycle test was used to assess maximal aerobic capacity (VO2max), as described previously (Kuipers et al., 1985). In addition, in vivo mitochondrial function in the knee extensor muscles was determined as phosphocreatine (PCrR) recovery rate, assessed by phosphorus magnetic resonance spectroscopy (31P-MRS) on a $3 \mathrm{~T}$ whole-body MRI scanner (Achieve 3T-X, Philips Healthcare), as previously described (Lindeboom et al., 2014).

\section{Plasma Analyses}

A fasted blood sample was drawn from the antecubital vein and collected in EDTA containing tubes. After centrifugation, the plasma was frozen in liquid nitrogen, followed by longterm storage at $-80^{\circ} \mathrm{C}$. Plasma glucose concentrations were determined using enzymatic assays on Cobas Bio Fara and Mira analyzers (hexokinase method [Roche, Basel, Switzerland]).

\section{Muscle Biopsies}

All muscle biopsies were collected after an overnight fast and in the basal state (i.e., before any intervention). Muscle specimens were taken from the $\mathrm{m}$. vastus lateralis under local anesthesia (2\% lidocaine) according to the technique of Bergstrom et al. (1967) and any visible non-muscle material was dissected from muscle tissue. Subsequently, biopsies were frozen immediately in melting isopentane cooled with liquid nitrogen and stored at $-80^{\circ} \mathrm{C}$ (Guo et al., 2001).

\section{miRNA Selection}

To identify miRNAs involved in insulin sensitivity a structured literature search was conducted between May 1st and May 24th 2016. The electronic database PubMed was consulted using the following search terms: "microRNA AND skeletal muscle AND insulin resistance OR microRNA AND skeletal muscle AND diabetes," which resulted in 44 original research articles (reviews were excluded). Articles were only considered for further analysis if an insulin resistant or diabetic model (cell, animal or human) was used, resulting in 11 remaining articles. Subsequently, miRNAs described in those 11 studies were only included in the current study if their significantly altered expression was confirmed by either RT-qPCR or northern blotting, or when miRNAs induced significant changes on any readout for insulin sensitivity after overexpression or knockdown. Finally, miRNAs were only included when full evolutionary conservation was observed in the online database miRbase, ultimately resulting in a list of 25 miRNAs.

\section{miRNA Isolation, cDNA Synthesis and miRNA RT-qPCR}

RNA extraction of the muscle biopsies was performed using the miRNeasy Mini kit (Qiagen, Germany). In a sterile glass tube, $700 \mu \mathrm{L}$ of Trizol was combined with $10-15 \mathrm{mg}$ of frozen muscle tissue and homogenized with an Ultra Turrax for $1 \mathrm{~min}$ at $17000 \mathrm{rpm}$. Total RNA isolation (including small RNAs) was performed according to the manufacturer's guidelines. RNA concentrations were measured on the nanodrop ND1000 (Biocompare, California, US) and quality was checked using a bioanalyzer (Agilent Technologies, California, US). All samples had RIN values between 7 and 10. Subsequently, RNA concentrations were diluted to a concentration of 5 $\mathrm{ng} / \mu \mathrm{l}$ with nuclease free water. The synthetic RNA spikein UniSp6 was added and cDNA was synthesized using the Universal cDNA Synthesis kit II (Exiqon, Denmark), according to the manufacturer's protocol. Isolated cDNA samples were analyzed by RT-PCR using predesigned 384-well Pick-\&-Mix microRNA PCR Panel plates (Exiqon, Denmark), according to the manufacturer's guidelines using a CFX384 Touch Realtime PCR detection system (Bio-Rad, the Netherlands). MiRNA expression data was normalized to the geometric mean of the expression of the miR-423-3p, SNORD48 and U6snRNA housekeeper genes.

\section{Statistics}

Subject characteristics and miRNA expression differences were assessed with the use of independent $t$-test to individually test the differences between the age- and BMI-matched subjects groups (i.e., T2DM patients vs. non-diabetic obese/overweight individuals and differences between lean sedentary individuals vs. endurance-trained athletes). Relationships between miRNA expression and peripheral insulin sensitivity were determined using Pearson correlation analysis. To test if confounding factors, such as age, BMI, fasting plasma glucose or fasting plasma insulin concentrations were contributing to the observed correlations, stepwise regression analysis was conducted using IBM SPSS Statistics for Windows (Version 24, Armonk, NY). Additionally, 
for each miRNA possible outliers were identified using the ROUT analysis (Motulsky and Brown, 2006), over the entire group of 48 subjects and using a cutoff value of $Q=1 \%$. A $P$-value below 0.05 was considered as significantly different.

\section{RESULTS}

\section{Selecting Candidate miRNAS}

Through a structured literature search and selection process, we selected miRNAs previously linked to insulin resistance or T2DM. This selection process resulted in 11 publications (Table 1) and 25 miRNAs (Table 2) meeting these criteria.

TABLE 1 | List of studies and miRNAs, previously demonstrated to be associated with skeletal muscle insulin sensitivity, that met the initial selection criteria of the current study.

\begin{tabular}{|c|c|c|c|c|}
\hline References & miRNA & Species & Validation & mirbase ID \\
\hline \multirow{3}{*}{$\begin{array}{l}\text { Zhou et al., } \\
2016\end{array}$} & Rno-miR-106b-5p & Rats & RT-qPCR & Hsa-miR-106b-5p \\
\hline & Rno-miR-30d-5p & & & Hsa-miR-30d-5p \\
\hline & Rno-miR-27a-3p & & & Hsa-miR-27a-3p \\
\hline $\begin{array}{l}\text { Latouche } \\
\text { et al., } 2016\end{array}$ & Rno-miR-194-5p & Rats & RT-qPCR & Hsa-miR-194-5p \\
\hline \multirow{2}{*}{$\begin{array}{l}\text { Bork-Jensen } \\
\text { et al., } 2015\end{array}$} & Hsa-miR-15b-5p & Human & Micro array & Hsa-miR-15b-5p \\
\hline & Hsa-miR-16-5p & & & Hsa-miR-16-5p \\
\hline \multirow{3}{*}{$\begin{array}{l}\text { Lee et al., } \\
2016\end{array}$} & Rno-miR-1-3p & Rats & RT-qPCR & Hsa-miR-1-3p \\
\hline & Rno-miR-16-5p & & & Hsa-miR-16-5p \\
\hline & Rno-miR-133b-3p & & & Hsa-miR-133b-3p \\
\hline \multirow{2}{*}{$\begin{array}{l}\text { Mohamed } \\
\text { et al., } 2014\end{array}$} & Mmu-miR-149-5p & Mice & RT-qPCR & Hsa-miR-149-5p \\
\hline & Mmu-miR-21a-5p & & & Hsa-miR-21-5p \\
\hline $\begin{array}{l}\text { Zhang et al., } \\
2015\end{array}$ & Mmu-miR-106b-5p & Mice & RT-qPCR & Hsa-miR-106b-5p \\
\hline $\begin{array}{l}\text { Agarwal et al., } \\
2013\end{array}$ & Mmu-miR-135a-5p & Mice & RT-qPCR & Hsa-miR-135a-5p \\
\hline \multirow{6}{*}{$\begin{array}{l}\text { Chen et al., } \\
2012\end{array}$} & Mmu-miR-1a-3p & Mice & RT-qPCR & Hsa-miR-1-3p \\
\hline & Mmu-miR-133a-5p & & & Hsa-miR-133a-3p \\
\hline & Mmu-miR-206-5p & & & Hsa-miR-206 \\
\hline & Mmu-miR-23b-3p & & & Hsa-miR-23b-3p \\
\hline & Mmu-miR-143-3p & & & Hsa-miR-143-3p \\
\hline & Mmu-miR-125a-3p & & & Hsa-miR-125a-3p \\
\hline \multirow{5}{*}{$\begin{array}{l}\text { Karolina et al., } \\
2011\end{array}$} & Rno-miR-144-3p & Rats & RT-qPCR & Hsa-miR-144-3p \\
\hline & Rno-miR-146a-5p & & & Hsa-miR-146a-5p \\
\hline & Rno-miR-150-5p & & & Hsa-miR-150-5p \\
\hline & Rno-miR-29a-3p & & & Hsa-miR-29a-3p \\
\hline & Rno-miR-192-5p & & & Hsa-miR-192-5p \\
\hline \multirow[t]{2}{*}{$\begin{array}{l}\text { Huang et al., } \\
2009\end{array}$} & Rno-miR-24-3p & Rats & $\begin{array}{l}\text { Northern } \\
\text { blot }\end{array}$ & Hsa-miR-24-3p \\
\hline & Rno-miR-126a-3p & & & Hsa-miR-126-3p \\
\hline \multirow[t]{3}{*}{$\begin{array}{l}\text { He et al., } \\
2007\end{array}$} & Rno-miR-29a-3p & Rats & $\begin{array}{l}\text { Northern } \\
\text { blot }\end{array}$ & Hsa-miR-29a-3p \\
\hline & Rno-miR-29b-3p & & & Hsa-miR-29b-3p \\
\hline & Rno-miR-29c-3p & & & Hsa-miR-29c-3p \\
\hline
\end{tabular}

\section{Basic Subject Characteristics}

The T2DM patients and the non-diabetic obese/overweight individuals as well as the lean sedentary individuals and the endurance-trained athletes were matched for age and BMI (Table 3). By design, the T2DM patients displayed increased fasting glucose levels $(7.9 \pm 0.5 \mathrm{mmol} / \mathrm{L})$ compared to nondiabetic obese/overweight individuals $(5.6 \pm 0.12 \mathrm{mmol} / \mathrm{L} ; p$ $<0.0001)$. Furthermore, fasting plasma glucose concentrations in sedentary individuals $(5.3 \pm 0.09 \mathrm{mmol} / \mathrm{L})$ and endurancetrained athletes $(5.1 \pm 0.08 \mathrm{mmol} / \mathrm{L})$ were similar (Table 3). Fasting plasma insulin was similar between the T2DM patients and non-diabetic obese/overweight individuals (20.5 \pm 2.5 vs. $21.7 \pm 3.8 \mu \mathrm{U} / \mathrm{mL}$ ) as well as between the lean sedentary individuals and endurance-trained athletes $(9.6 \pm 1.4$ vs. $7.0 \pm$ $0.8 \mu \mathrm{U} / \mathrm{mL}$ ) (Table 3). Body fat percentage of T2DM patients and non-diabetic obese/overweight individuals was also similar $(34.7 \pm 1.6$ vs. $34.7 \pm 2.0 \%)$ but was lower in endurance-trained athletes compared to lean sedentary individuals $(12.8 \pm 1.1$ vs. $18.3 \pm 1.1 \% ; p<0.0001$; Table 3).

\section{Peripheral Insulin Sensitivity}

As anticipated, peripheral insulin sensitivity, i.e., the glucose infusion rate, normalized to body weight, required to maintain euglycemia during the hyperinsulinemic-euglycemic clamp,

TABLE 2 | Final list of all miRNA candidates included in the present study, based on a comprehensive PubMed-based literature search.

\begin{tabular}{lll}
\hline microRNAs & miR base IDs & Mature sequences (5'-3') $^{\prime}$ \\
\hline hsa-miR-1-3p & MIMAT0000416 & UGGAAUGUAAAGAAGUAUGUAU \\
hsa-miR-106b-5p & MIMAT0000680 & UAAAGUGCUGACAGUGCAGAU \\
hsa-miR125a-3p & MIMAT0004602 & ACAGGUGAGGUUCUUGGGAGCC \\
hsa-mir-126-3p & MIMAT0000445 & UCGUACCGUGAGUAAUAAUGCG \\
hsa-miR-133a-3p & MIMAT0000427 & UUUGGUCCCCUUCAACCAGCUG \\
hsa-miR-133b-3p & MIMAT0000770 & UUUGGUCCCCUUCAACCAGCUA \\
hsa-miR-135a-5p & MIMAT0000428 & UAUGGCUUUUUAUUCCUAUGUGA \\
hsa-miR-143-3p & MIMAT0000435 & UGAGAUGAAGCACUGUAGCUC \\
hsa-miR-144-3p & MIMAT0000436 & UACAGUAUAGAUGAUGUACU \\
hsa-miR-146a-5p & MIMAT0000449 & UGAGAACUGAAUUCCAUGGGUU \\
hsa-miR-149-5p & MIMAT0000450 & UCUGGCUCCGUGUCUUCACUCCC \\
hsa-miR-150-5p & MIMAT0000451 & UCUCCCAACCCUUGUACCAGUG \\
hsa-miR-15b-5p & MIMAT0000417 & UAGCAGCACAUCAUGGUUUACA \\
hsa-miR-16-5p & MIMAT0000069 & UAGCAGCACGUAAAUAUUGGCG \\
hsa-miR-192-5p & MIMAT0000222 & CUGACCUAUGAAUGACAGCC \\
hsa-miR-194-5p & MIMAT0000460 & UGUAACAGCAACUCCAUGUGGA \\
hsa-miR-206 & MIMAT0000462 & UGGAAUGUAAGGAAGUGUGUGG \\
hsa-miR-21-5p & MIMAT0000076 & UAGCUUAUCAGACUGAUGUUGA \\
hsa-miR-23b-3p & MIMAT0000418 & AUCACAUUGCCAGGGAUUACC \\
hsa-miR-24-3p & MIMAT0000080 & UGGCUCAGUUCAGCAGGAACAG \\
hsa-miR-27a-3p & MIMAT0000084 & UUCACAGUGGCUAAGUUCCGC \\
hsa-miR-29a-3p & MIMAT0000086 & UAGCACCAUCUGAAAUCGGUUA \\
hsa-miR-29b-3p & MIMAT0000100 & UAGCACCAUUUGAAAUCAGUGUU \\
hsa-miR-29c-3p & MIMAT0000681 & UAGCACCAUUUGAAAUCGGUUA \\
hsa-miR-30d-5p & MIMAT0000245 & UGUAAACAUCCCCGACUGGAAG \\
& &
\end{tabular}


TABLE 3 | Subject characteristics.

\begin{tabular}{|c|c|c|c|c|c|c|}
\hline & T2DM & Obese & $p$-value & Lean & Athletes & $p$-value \\
\hline Subjects & 12 & 12 & & 12 & 12 & \\
\hline Age (yrs) & $58.83 \pm 1.13$ & $56.67 \pm 2.09$ & 0.45 & $22.25 \pm 0.74$ & $25.08 \pm 1.25$ & 0.05 \\
\hline Height (m) & $1.76 \pm 2.25$ & $1.74 \pm 2.16$ & 0.24 & $1.83 \pm 1.60$ & $1.83 \pm 2.15$ & 0.90 \\
\hline Weight (kg) & $101 \pm 3.68$ & $94.1 \pm 3.99$ & 0.22 & $73.4 \pm 1.98$ & $70.33 \pm 2.14$ & 0.31 \\
\hline $\mathrm{BMI}\left(\mathrm{kg} / \mathrm{m}^{2}\right)$ & $32.44 \pm 1.02$ & $31.83 \pm 1.06$ & 0.34 & $22.02 \pm 0.55$ & $20.98 \pm 0.44$ & 0.20 \\
\hline Body fat (\%) & $34.69 \pm 1.63$ & $34.65 \pm 2.01$ & 0.97 & $18.34 \pm 1.05$ & $12.76 \pm 0.60$ & $<0.0001$ \\
\hline Lean body mass (kg) & $65.79 \pm 1.87$ & $60.95 \pm 2.59$ & 0.09 & $57.77 \pm 1.78$ & $58.98 \pm 1.64$ & 0.62 \\
\hline FPG (mmol/L) & $7.92 \pm 0.48$ & $5.64 \pm 0.12$ & 0.0001 & $5.25 \pm 0.09$ & $5.13 \pm 0.08$ & 0.30 \\
\hline FPI $(\mu \mathrm{U} / \mathrm{mL})$ & $20.53 \pm 2.51$ & $21.74 \pm 3.83$ & 0.79 & $9.59 \pm 1.36$ & $6.98 \pm 0.75$ & 0.10 \\
\hline HbA1c (\%) & $7.18 \pm 0.22$ & $5.71 \pm 0.10$ & $<0.0001$ & - & - & - \\
\hline Triacylglycerol (mmol/L) & $2.30 \pm 0.32$ & $1.31 \pm 0.09$ & 0.02 & - & - & - \\
\hline HDL (mmol/L) & $1.03 \pm 0.09$ & $1.60 \pm 0.40$ & 0.18 & - & - & - \\
\hline LDL (mmol/L) & $2.69 \pm 0.12$ & $3.12 \pm 0.31$ & 0.28 & - & - & - \\
\hline FFA (mmol/L) & $0.58 \pm 0.05$ & $0.69 \pm 0.14$ & 0.49 & - & - & - \\
\hline PCr Recovery (s) & $26.54 \pm 1.69$ & $21.26 \pm 1.28$ & 0.03 & $20.22 \pm 1.44$ & $15.58 \pm 1.56$ & 0.07 \\
\hline $\mathrm{VO}_{2} \max (\mathrm{ml} / \mathrm{min} / \mathrm{kg})$ & $24.51 \pm 1.13$ & $27.81 \pm 1.25$ & 0.08 & $41.36 \pm 0.57$ & $59.97 \pm 1.20$ & $<0.0001$ \\
\hline
\end{tabular}

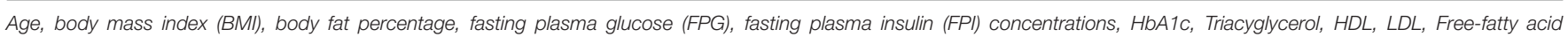

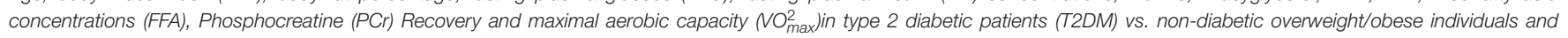
lean sedentary individuals vs. endurance-trained athletes. Values presented are mean \pm SEM.

was significantly lower in T2DM patients compared to nondiabetic obese/overweight individuals (14.5 \pm 2.0 vs. $26.6 \pm$ $2.5 \mu \mathrm{mol} / \mathrm{min} / \mathrm{kg}$, respectively; $p=0.0011)$. In turn, insulin sensitivity was significantly higher in endurance-trained athletes as compared to lean sedentary individuals $(76.5 \pm 4.6$ vs. $54.9 \pm$ $3.1 \mu \mathrm{mol} / \mathrm{min} / \mathrm{kg}$, respectively; $p<0.0001$; Figure 1A). Similar results were obtained when GIR was normalized to lean body mass (Figure 1B).

\section{miRNAs Differentially Expressed between Type 2 Diabetic Patients and Non-diabetic Obese/Overweight Individuals}

To determine which miRNAs were differentially expressed during T2DM, we compared their relative expression levels observed in T2DM patients with age- and BMI-matched nondiabetic obese/overweight individuals (Table 4). Interestingly, out of 25 miRNAs, selected based on previously reported links with insulin sensitivity, only 6 miRNAs were differentially expressed in T2DM, all of which were upregulated in T2DM patients compared to non-diabetic obese/overweight individuals (Figure 2). Thus, miRNA-133b-3p (1.00 \pm 0.04 vs. $0.83 \pm 0.05 ; p$ $=0.02)$, miRNA-206 (2.04 \pm 0.23 vs. $1.18 \pm 0.11 ; p=0.001)$, and miRNA-27a-3p (1.07 \pm 0.05 vs. $0.93 \pm 0.04 ; p=0.04)$ were all significantly higher in T2DM patients vs. non-diabetic obese/overweight individuals, respectively. Thus, the expression of miRNA-29a-3p (1.05 \pm 0.07 vs. $0.86 \pm 0.05 ; p=0.03)$, miRNA$29 b-3 p(1.50 \pm 0.12$ vs. $1.16 \pm 0.08 ; p=0.02)$, and miRNA$29 \mathrm{c}-3 \mathrm{p}$ ( $1.20 \pm 0.06$ vs. $1.00 \pm 0.06 ; p=0.02)$ were also higher in T2DM patients vs. non-diabetic obese/overweight individuals, respectively (Figure 2 ).

When comparing miRNA expression levels between lean sedentary individuals and endurance-trained athletes, we found miRNA126-3p $(1.16 \pm 0.06$ vs. $0.88 \pm 0.03 ; p<0.001)$ and

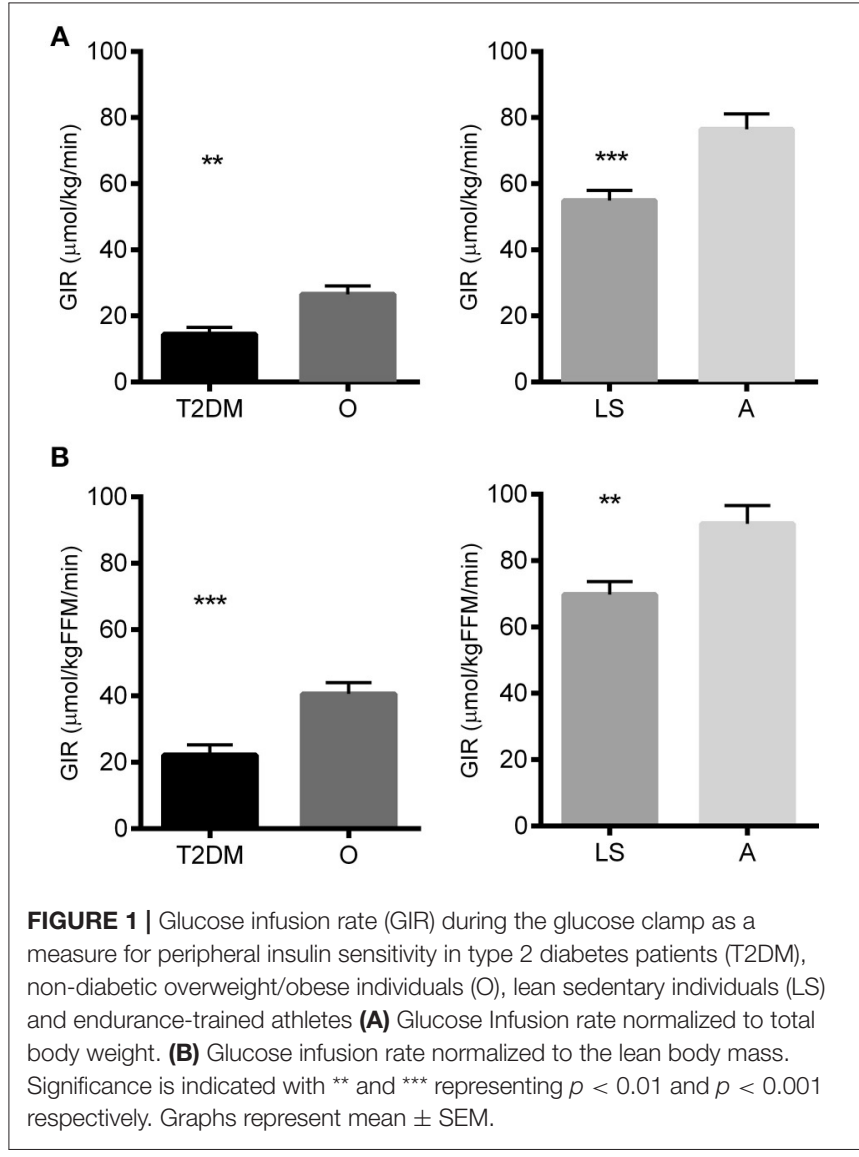

miRNA-150-5p (1.14 \pm 0.07 vs. $0.90 \pm 0.05 ; p=0.01)$ to be upregulated in endurance-trained athletes compared to lean sedentary individuals (Table 4). 
TABLE 4 | List of relative miRNA expressions in type 2 diabetic patients (T2DM) vs. non-diabetic overweight/obese individuals and lean sedentary individuals vs. endurance-trained athletes.

\begin{tabular}{|c|c|c|c|c|c|c|}
\hline miRNA & T2DM & Obese & $p$-value & Lean sedentary & Athletes & $p$-value \\
\hline hsa-miR-1-3p & $0.93 \pm 0.07$ & $0.85 \pm 0.05$ & 0.34 & $1.05 \pm 0.06$ & $0.87 \pm 0.03$ & 0.05 \\
\hline hsa-miR-106b-5p & $1.56 \pm 0.32$ & $1.42 \pm 0.21$ & 0.85 & $1.00 \pm 0.10$ & $0.80 \pm 0.08$ & 0.16 \\
\hline hsa-miR-125a-3p & $0.97 \pm 0.15$ & $0.68 \pm 0.09$ & 0.11 & $0.78 \pm 0.10$ & $1.15 \pm 0.15$ & 0.06 \\
\hline hsa-miR-126-3p & $0.83 \pm 0.06$ & $0.71 \pm 0.04$ & 0.12 & $0.88 \pm 0.03$ & $1.16 \pm 0.06$ & 0.001 \\
\hline hsa-miR-133a-3p & $0.96 \pm 0.07$ & $0.84 \pm 0.04$ & 0.24 & $0.94 \pm 0.05$ & $0.81 \pm 0.03$ & 0.03 \\
\hline hsa-miR-133b-3p & $1.00 \pm 0.04$ & $0.83 \pm 0.05$ & 0.02 & $0.94 \pm 0.05$ & $0.83 \pm 0.03$ & 0.05 \\
\hline hsa-miR-135a-5p & $0.52 \pm 0.09$ & $0.44 \pm 0.08$ & 0.88 & $0.44 \pm 0.10$ & $0.69 \pm 0.14$ & 0.16 \\
\hline hsa-miR-143-3p & $1.21 \pm 0.36$ & $0.83 \pm 0.08$ & 0.44 & $0.86 \pm 0.09$ & $0.94 \pm 0.06$ & 0.48 \\
\hline hsa-miR-144-3p & $1.14 \pm 0.31$ & $1.11 \pm 0.23$ & 0.57 & $0.77 \pm 0.14$ & $0.44 \pm 0.09$ & 0.06 \\
\hline hsa-miR-146a-5p & $1.78 \pm 0.35$ & $1.45 \pm 0.15$ & 0.98 & $1.17 \pm 0.14$ & $1.02 \pm 0.17$ & 0.08 \\
\hline hsa-miR-149-5p & $1.43 \pm 0.09$ & $1.50 \pm 0.11$ & 0.66 & $1.34 \pm 0.09$ & $1.28 \pm 0.13$ & 0.28 \\
\hline hsa-miR-150-5p & $0.85 \pm 0.08$ & $0.71 \pm 0.04$ & 0.16 & $0.90 \pm 0.05$ & $1.14 \pm 0.07$ & 0.01 \\
\hline hsa-miR-15b-5p & $1.36 \pm 0.29$ & $1.33 \pm 0.24$ & 0.51 & $0.94 \pm 0.13$ & $0.67 \pm 0.09$ & 0.10 \\
\hline hsa-miR-16-5p & $1.30 \pm 0.27$ & $1.17 \pm 0.19$ & 0.38 & $0.80 \pm 0.10$ & $0.52 \pm 0.06$ & 0.05 \\
\hline hsa-miR-192-5p & $1.48 \pm 0.28$ & $1.38 \pm 0.16$ & 0.77 & $1.09 \pm 0.11$ & $1.09 \pm 0.11$ & 0.53 \\
\hline hsa-miR-194-5p & $1.62 \pm 0.38$ & $1.46 \pm 0.25$ & 0.91 & $1.07 \pm 0.12$ & $1.01 \pm 0.12$ & 0.72 \\
\hline hsa-miR-206 & $2.04 \pm 0.23$ & $1.18 \pm 0.12$ & 0.003 & $1.18 \pm 0.11$ & $0.77 \pm 0.09$ & 0.009 \\
\hline hsa-miR-21-5p & $1.66 \pm 0.36$ & $1.06 \pm 0.06$ & 0.23 & $1.03 \pm 0.06$ & $1.01 \pm 0.07$ & 0.82 \\
\hline hsa-miR-23b-3p & $1.04 \pm 0.05$ & $0.92 \pm 0.04$ & 0.10 & $0.86 \pm 0.03$ & $0.98 \pm 0.03$ & 0.03 \\
\hline hsa-miR-24-3p & $1.11 \pm 0.06$ & $0.99 \pm 0.05$ & 0.11 & $0.97 \pm 0.04$ & $1.03 \pm 0.05$ & 0.43 \\
\hline hsa-miR-27a-3p & $1.07 \pm 0.05$ & $0.93 \pm 0.04$ & 0.04 & $0.89 \pm 0.03$ & $0.88 \pm 0.04$ & 0.97 \\
\hline hsa-miR-29a-3p & $1.05 \pm 0.07$ & $0.86 \pm 0.05$ & 0.03 & $0.87 \pm 0.04$ & $0.86 \pm 0.03$ & 0.77 \\
\hline hsa-miR-29b-3p & $1.50 \pm 0.12$ & $1.16 \pm 0.08$ & 0.02 & $0.89 \pm 0.03$ & $0.82 \pm 0.04$ & 0.25 \\
\hline hsa-miR-29c-3p & $1.2 \pm 0.06$ & $1.00 \pm 0.06$ & 0.02 & $0.99 \pm 0.03$ & $0.91 \pm 0.04$ & 0.13 \\
\hline hsa-miR-30d-5p & $0.76 \pm 0.05$ & $0.67 \pm 0.03$ & 0.15 & $0.78 \pm 0.04$ & $0.79 \pm 0.03$ & 0.96 \\
\hline
\end{tabular}

Values presented are mean \pm SEM.

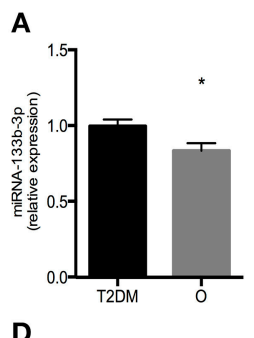

D

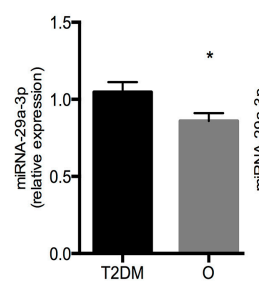

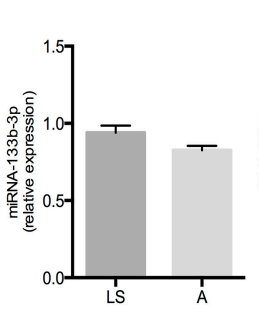

B

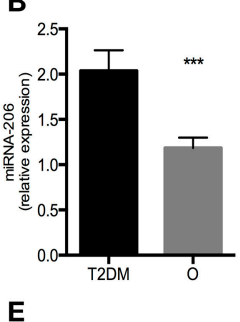

E
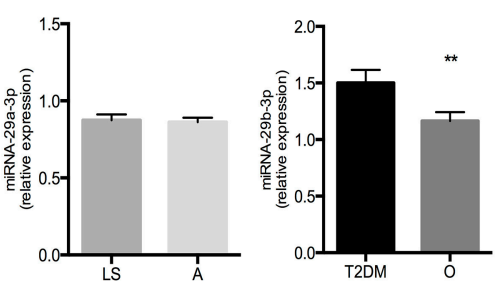
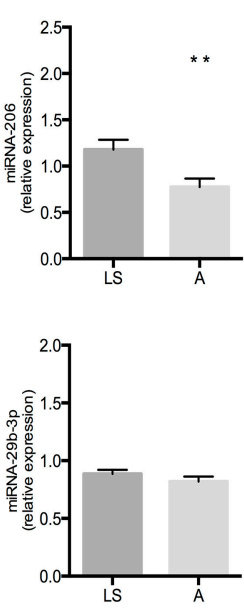
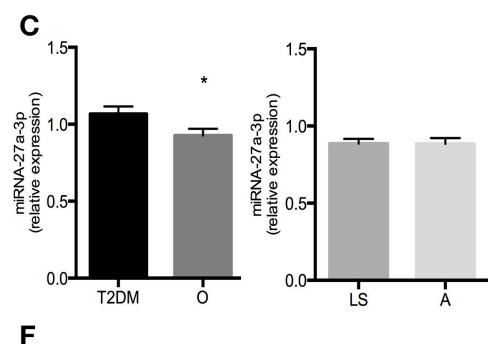

$F$
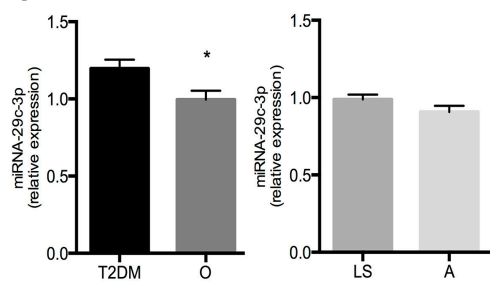

FIGURE 2 | Relative expression levels of miR-133b-3p (A), miR-206-5p (B), miR-27a-3p (C), miR-29a-3p (D), miR-29b-3p (E), and miR-29c-3p (F) in type 2 diabetic subjects (T2DM) vs. non-diabetic overweight/obese individuals $(\mathrm{O})$ and lean sedentary individuals (LS) vs. endurance-trained athletes (A). Significance is indicated with ${ }^{*},{ }^{* *}$, and ${ }^{* * *}$ representing $p<0.05, p<0.01$, and $p<0.001$, respectively. Graphs represent mean \pm SEM. 


\section{miRNA-27a-3p and the miRNA-29 Family Relate to in Vivo Peripheral Insulin \\ Sensitivity}

In order to determine whether the 6 differentially expressed miRNAs in T2DM patients (relative to obese/overweight controls) also are associated with peripheral insulin sensitivity, we correlated their expression levels to the glucose infusion rate (GIR) during the hyperinsulinemic-euglycemic clamp (Figure 3A). Five miRNAs displayed a significant and negative correlation between the relative miRNA expression and the GIR during the glucose clamp (Figure 3A). The correlation between the expression of miRNA-133b-3p and GIR just did not reach significance $(r=-0.28, p=0.06)$. In order to visualize inter- and intra-group variation of the miRNA expressions and GIR, the correlations were also displayed per group (Figure 3B).

Our four subject groups not only differ in peripheral insulin sensitivity, but also in other subject characteristics, such as age, BMI and mitochondrial capacity (Supplemental Figure 1). To explore if the correlations of these 6 miRNAs with insulin sensitivity could also be explained by other factors we performed a stepwise linear regression analysis including VO2max, phosphocreatine recovery rate (PCrR), age, BMI, body

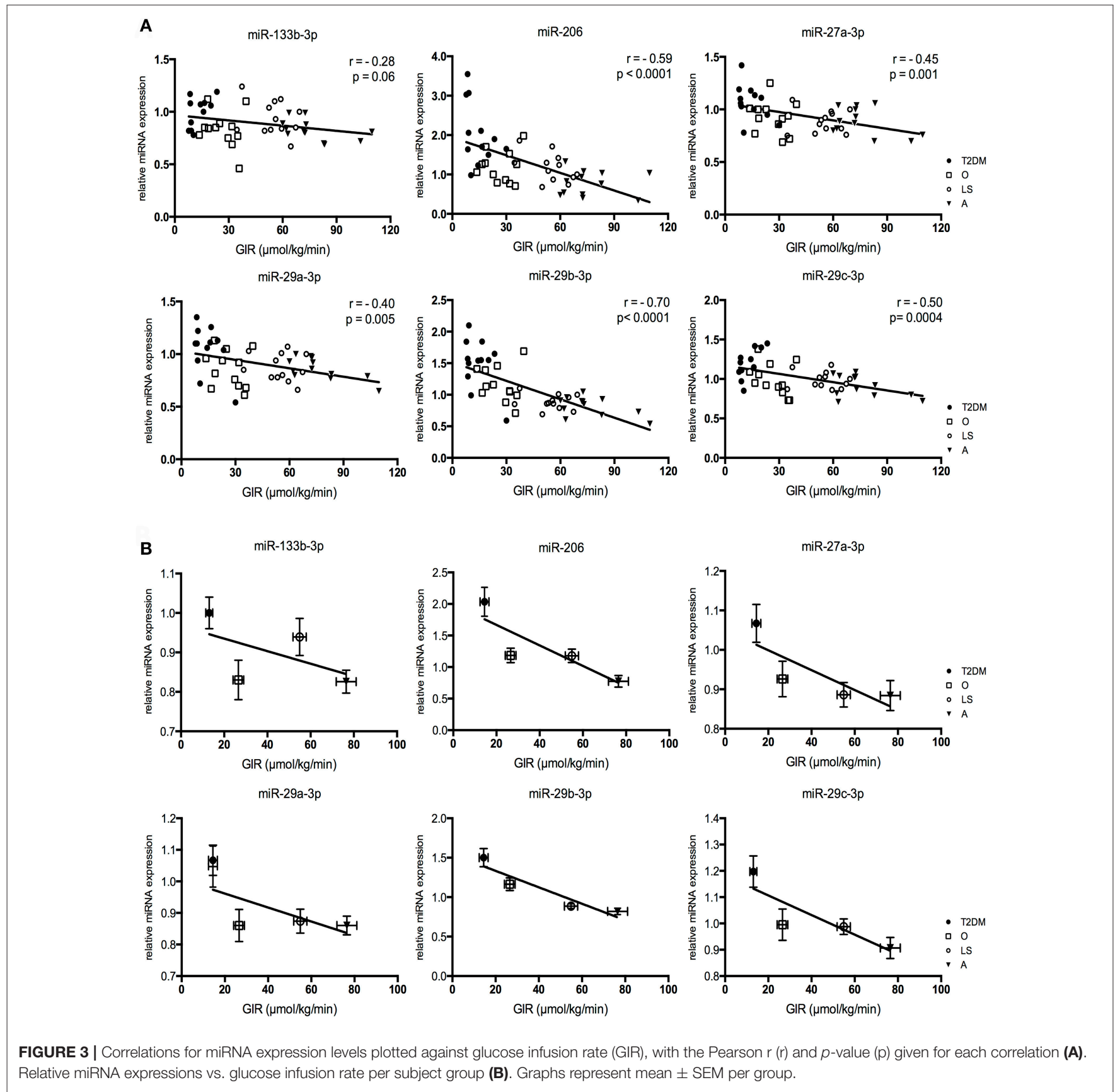


fat percentage, fasting plasma glucose (FPG) concentrations, fasting plasma insulin (FPI) concentrations and GIR as possible determinants for miRNA expression. This analysis revealed that for miRNA-27-3p (included variable: GIR, $p=0.004$; excluded variables: $\mathrm{BMI}, p=0.23$; VO2max, $p=0.88$; PCrR, $p=0.45$; FPG, $p=0.18$; FPI, $p=0.96$; age, $p=0.36$; fat $\%, p=0.93$ ), miRNA29a-3p (included variable: GIR, $p=0.002$; excluded variables: BMI, $p=0.37$; VO2max, $p=0.26$; PCrR, $p=0.36$; FPG, $p=$ 0.22 ; FPI, $p=0.44$; age, $p=1.0$; fat $\%, p=0.40$ ), miRNA-29b$3 p$ (included variable: GIR, $p=0.0001$; excluded variables: $\mathrm{BMI}$, $p=0.81$; VO2max, $p=0.76$; PCrR, $p=0.22$; FPG, $p=0.10$; FPI, $p=0.87$; age, $p=0.07$; fat $\%, p=0.37$ ) and miRNA-29c$3 \mathrm{p}$ (included variable: GIR, $p=0.0001$; excluded variables: $\mathrm{BMI}$, $p=0.58$; VO2max, $p=0.36$; PCrR, $p=0.64$; FPG, $p=0.37$; FPI, $p=0.56$; age, $p=0.97$; fat $\%, p=0.51$ ) all variables other than GIR were excluded from the model, further illustrating the relation between these miRNAs and peripheral insulin sensitivity. For miRNA-206, all variables but PCrR and FPI were excluded from the model (Included variable: PCrR, $p=0.001$ and FPI, $p$ $=0.003$; excluded variables: $\mathrm{BMI}, p=0.57$; VO2 $\max , p=0.89$; FPG, $p=0.18$; age, $p=0.65$; fat $\%, p=0.84$ ), indicating that the expression of miRNA-206 is associated with mitochondrial capacity and fasting plasma insulin concentrations.

\section{DISCUSSION}

MiRNAs have been reported to be involved in insulin sensitivity but information is primarily derived from preclinical cell- and animal models for insulin resistance. In the present study, we investigated 25 miRNAs, which have previously been associated with insulin sensitivity. We examined the expression of these miRNAs in subjects possessing a wide range in peripheral insulin sensitivity. We identified miRNA-27a-3p and all three members of the miRNA-29 family to be differentially regulated in muscle from patients with T2DM as compared to normoglycemic obese/overweight individuals. The expression of these miRNAs were significantly and strongly correlated to insulin sensitivity in human skeletal muscle.

Here, we show that, out of the 25 selected miRNAs, 6 candidate miRNAs (miRNA-133b-3p, miRNA-206, miRNA27a-3p, miRNA-29a-3p, miRNA-29b-3p, and miRNA-29c-3p) were differently expressed in T2DM patients vs. non-diabetic overweight/obese individuals (Figure 2). Subsequent stepwise regression analysis of the relative miRNA expression levels and peripheral insulin sensitivity, supported the notion that these associations of miRNA-27a-3p, miRNA-29a-3p, miRNA-29b$3 p$ and miRNA-29c-3p with peripheral insulin sensitivity were rather direct associations which were not confounded by cocorrelations with other variables (i.e., VO2max, PCrR, age, BMI, body fat percentage, FPG, and FPI) that were (by design) different between these 4 phenotypically different groups (Figure 3 ).

The myomiR miRNA-206 was selected since it was previously reported to be downregulated in gastrocnemius muscle of high-fat diet-induced insulin resistant mice (Chen et al., 2012). In contrast to these findings, we show here that miRNA-206 expression was increased in skeletal muscle of
T2DM patients as compared to non-diabetic obese/overweight individuals (Figure 2), and that miRNA-206 correlated negatively with peripheral insulin sensitivity (Figure 3). Besides obvious differences in species, it should also be noted that all subjects included in this study were weight stable for at least 6 months whereas the mice displaying differences in gene expression of miRNA-206, were in a positive energy balance and were gaining weight on a high-fat diet significantly throughout the study. Nonetheless, a possible mechanism linking miRNA-206 to insulin sensitivity is provided by a study demonstrating that reduced miRNA-206 levels increases IGF1 mRNA levels in vivo (Yan et al., 2013). IGF-1 has been demonstrated to stimulate insulin-like actions in vitro, including glucose transport, oxidation and GLUT4 translocation (Dimitriadis et al., 1992). These findings suggest that the increased miRNA-206 expression levels we observed in T2DM patients may mediate insulin resistance by inhibiting IGF-1 expression. However, although miRNA-206 expression levels were different between T2DM patients and non-diabetic obese individuals, regression analysis revealed that the observed relationship between peripheral insulin sensitivity and miRNA-206 expression was primarily confounded by in vivo mitochondrial capacity and fasting plasma insulin concentrations. Interestingly, a previous study in human subjects indeed reported decreased skeletal muscle miRNA-206 expression upon 12-weeks of endurance training, which was accompanied by increases in mitochondrial capacity and insulin sensitivity (Nielsen et al., 2010), indeed pointing toward a role for miRNA-206 in the regulation of skeletal muscle mitochondrial oxidative capacity, a factor that has been repeatedly demonstrated for its involvement in insulin sensitivity (Hoeks and Schrauwen, 2012).

Previous research revealed that miRNA-27a-3p was markedly elevated in a rat model of T2DM (Zhou et al., 2016). In line with these findings, we here observed increased miRNA-27a$3 \mathrm{p}$ levels in skeletal muscle of T2DM patients as compared to non-diabetic overweight/obese individuals. In addition, we demonstrated a strong negative correlation between miRNA27a-3p expression and peripheral insulin sensitivity. Additional support for the involvement of miRNA-27a-3p in the regulation of insulin sensitivity comes from in vitro studies in L6 muscle cells showing that miRNA-27a-3p knockdown improved insulin signaling and glucose uptake in insulin-induced insulin-resistant L6 muscle cells (Zhou et al., 2016). Furthermore, a cohort study of 163 T2DM patients and 185 healthy controls revealed that the presence of a SNP in the sequence of miRNA-27a, resulting in compromised gene expression levels, was associated with reduced risk for T2DM susceptibility (Ciccacci et al., 2013).

Finally, all members of the miRNA-29 family have previously been reported to be upregulated in several tissues, including skeletal muscle, derived from various rat models of T2DM (He et al., 2007; Karolina et al., 2011). In agreement with these findings, the present study also indicates that all three members of the miRNA-29 family (miRNA-29a-3p, miRNA-29b-3p and miRNA-29c-3p) are upregulated in skeletal muscle tissue of T2DM patients (Figure 1). Moreover, we demonstrate that all three miRNA-29 members also significantly and negatively correlated with peripheral insulin sensitivity 
(Figure 3), supporting recent findings in human (Massart et al., 2017). Previous research also demonstrated increased expression of miRNA-29 family members in whole blood of T2DM patients, as compared to healthy, normoglycemic controls (Karolina et al., 2011; Kong et al., 2011). Furthermore, overexpression of the miRNA-29 family in 3T3-L1 adipocytes resulted in an impaired insulin-stimulated glucose uptake and intracellular insulin signaling, whereas incubation of adipocytes with high insulin and glucose levels resulted in an upregulation of miRNA29a and miRNA-29b (He et al., 2007). Mechanistically, the actions of the miRNA-29 family may be mediated via effects on IGF1 (Smith et al., 2013), AKT2 (Karolina et al., 2011), Caveolin2 (Cav2) and syntaxin-1, all molecules implicated in insulin stimulated glucose uptake in muscle cells (Oh et al., 2006) or GLUT4 vesicle-membrane interactions, respectively (Dulubova et al., 1999; Macaulay et al., 2002; He et al., 2007). Interestingly, the miR-29 family members all present a predicted binding site on at least one of the $3^{\prime}$ UTR messenger RNAs described above.

In conclusion, we investigated 25 miRNAs for which previous, preclinical research indicated an association with insulin sensitivity, and we quantified their expression levels in skeletal muscle biopsies derived from, T2DM patients, nondiabetic overweight/obese individuals, lean sedentary individuals and endurance-trained athletes, hence representing a wide range in peripheral insulin sensitivity. We demonstrate that miRNA27a-3p and all three members of the miRNA-29 family were upregulated in skeletal muscle of T2DM patients compared to non-diabetic overweight/obese individuals, and additionally

\section{REFERENCES}

Agarwal, P., Srivastava, R., Srivastava, A. K., Ali, S., and Datta, M. (2013). miR-135a targets IRS2 and regulates insulin signaling and glucose uptake in the diabetic gastrocnemius skeletal muscle. Biochim. Biophys. Acta 1832, 1294-1303. doi: 10.1016/j.bbadis.2013.03.021

Bartel, D. P. (2004). MicroRNAs: genomics, biogenesis, mechanism, and function. Cell 116, 281-297. doi: 10.1016/S0092-8674(04)00045-5

Bartel, D. P. (2009). MicroRNAs: target recognition and regulatory functions. Cell 136, 215-233. doi: 10.1016/j.cell.2009.01.002

Bergstrom, J., Hermansen, L., Hultman, E., and Saltin, B. (1967). Diet, muscle glycogen and physical performance. Acta Physiol. Scand. 71, 140-150. doi: 10.1111/j.1748-1716.1967.tb03720.x

Bork-Jensen, J., Scheele, C., Christophersen, D. V., Nilsson, E., Friedrichsen, M., Fernandez-Twinn, D. S., et al. (2015). Glucose tolerance is associated with differential expression of microRNAs in skeletal muscle: results from studies of twins with and without type 2 diabetes. Diabetologia 58, 363-373. doi: 10.1007/s00125-014-3434-2

Chen, G. Q., Lian, W. J., Wang, G. M., Wang, S., Yang, Y. Q., and Zhao, Z. W. (2012). Altered microRNA expression in skeletal muscle results from highfat diet-induced insulin resistance in mice. Mol. Med. Rep. 5, 1362-1368. doi: $10.3892 / \mathrm{mmr} .2012 .824$

Ciccacci, C., Di Fusco, D., Cacciotti, L., Morganti, R., D’Amato, C., Greco, C., et al. (2013). MicroRNA genetic variations: association with type 2 diabetes. Acta Diabetol. 50, 867-872. doi: 10.1007/s00592-013-0469-7

Dahlmans, D., Houzelle, A., Schrauwen, P., and Hoeks, J. (2016). Mitochondrial dynamics, quality control and miRNA regulation in skeletal muscle: implications for obesity and related metabolic disease. Clin. Sci. 130, 843-852. doi: 10.1042/CS20150780 displayed strong negative correlations with peripheral insulin sensitivity across the four metabolically distinct human subject groups. These miRNAs may therefore hold potential as novel targets in the modulation of insulin resistance.

\section{AUTHOR CONTRIBUTIONS}

DD designed and performed the experiments, analyzed data, and wrote the manuscript. AH and JJ designed and performed the experiments and assisted in the analysis. EP, LL, and $\mathrm{MH}$ contributed to the initial aspects of study design and assisted in the analysis. PS and JH contributed to the design of the study, analyzed and interpreted the data and reviewed and edited the manuscript. All authors reviewed and approved the final version of the manuscript.

\section{ACKNOWLEDGMENTS}

This work was supported by a VIDI (917.14.358 to JH) and a VICI (918.96.618 to PS) grant from the Netherlands Organization for Scientific Research (NWO), respectively, and a grant from the Dutch Diabetes Research Foundation (grant 2013.82.1639 to JH).

\section{SUPPLEMENTARY MATERIAL}

The Supplementary Material for this article can be found online at: http://journal.frontiersin.org/article/10.3389/fphys. 2017.00711/full\#supplementary-material

DeFronzo, R. A., Gunnarsson, R., Bjorkman, O., Olsson, M., and Wahren, J. (1985). Effects of insulin on peripheral and splanchnic glucose metabolism in noninsulin-dependent (type II) diabetes mellitus. J. Clin. Invest. 76, 149-155. doi: 10.1172/JCI111938

DeFronzo, R. A., Tobin, J. D., and Andres, R. (1979). Glucose clamp technique: a method for quantifying insulin secretion and resistance. Am. J. Physiol. 237, E214-223.

Dimitriadis, G., Parry-Billings, M., Bevan, S., Dunger, D., Piva, T., Krause, U., et al. (1992). Effects of insulin-like growth factor I on the rates of glucose transport and utilization in rat skeletal muscle in vitro. Biochem. J. 285(Pt 1), 269-274.

Dulubova, I., Sugita, S., Hill, S., Hosaka, M., Fernandez, I., Sudhof, T. C., et al. (1999). A conformational switch in syntaxin during exocytosis: role of munc18. EMBO J. 18, 4372-4382. doi: 10.1093/emboj/18.16.4372

Guo, Z., Mishra, P., and Macura, S. (2001). Sampling the intramyocellular triglycerides from skeletal muscle. J. Lipid Res. 42, 1041-1048.

He, A., Zhu, L., Gupta, N., Chang, Y., and Fang, F. (2007). Overexpression of micro ribonucleic acid 29, highly up-regulated in diabetic rats, leads to insulin resistance in 3T3-L1 adipocytes. Mol. Endocrinol. 21, 2785-2794. doi: 10.1210/me.2007-0167

Hoeks, J., and Schrauwen, P. (2012). Muscle mitochondria and insulin resistance: a human perspective. Trends Endocrinol. Metab. 23, 444-450. doi: 10.1016/j.tem.2012.05.007

Huang, B., Qin, W., Zhao, B., Shi, Y., Yao, C., Li, J., et al. (2009). MicroRNA expression profiling in diabetic GK rat model. Acta Biochim. Biophys. Sin. (Shanghai) 41, 472-477. doi: 10.1093/abbs/gmp035

Karolina, D. S., Armugam, A., Tavintharan, S., Wong, M. T., Lim, S. C., Sum, C. F., et al. (2011). MicroRNA 144 impairs insulin signaling by inhibiting the expression of insulin receptor substrate 1 in type 2 diabetes mellitus. PLoS ONE 6:e22839. doi: 10.1371/annotation/698b7123-174f-4a09-95c9-fd6f5017d622 
Kong, L., Zhu, J., Han, W., Jiang, X., Xu, M., Zhao, Y., et al. (2011). Significance of serum microRNAs in pre-diabetes and newly diagnosed type 2 diabetes: a clinical study. Acta Diabetol. 48, 61-69. doi: 10.1007/s00592-010-0226-0

Krutzfeldt, J., and Stoffel, M. (2006). MicroRNAs: a new class of regulatory genes affecting metabolism. Cell Metab. 4, 9-12. doi: 10.1016/j.cmet.2006.05.009

Kuipers, H., Verstappen, F. T., Keizer, H. A., Geurten, P., and van Kranenburg, G. (1985). Variability of aerobic performance in the laboratory and its physiologic correlates. Int. J. Sports Med. 6, 197-201. doi: 10.1055/s-2008-1025839

Latouche, C., Natoli, A., Reddy-Luthmoodoo, M., Heywood, S. E., Armitage, J. A., and Kingwell, B. A. (2016). MicroRNA-194 modulates glucose metabolism and its skeletal muscle expression is reduced in diabetes. PLOS ONE 11:e0155108. doi: 10.1371/journal.pone.0155108

Lee, D. E., Brown, J. L., Rosa, M. E., Brown, L. A., Perry, R. A. Jr., Wiggs, M. P., et al. (2016). MicroRNA-16 is downregulated during insulin resistance and controls skeletal muscle protein accretion. J. Cell. Biochem. 117, 1775-1787. doi: $10.1002 /$ jcb. 25476

Lindeboom, L., Nabuurs, C. I., Hoeks, J., Brouwers, B., Phielix, E., Kooi, M. E., et al. (2014). Long-echo time MR spectroscopy for skeletal muscle acetylcarnitine detection. J. Clin. Invest. 124, 4915-4925. doi: 10.1172/JCI74830

Macaulay, S. L., Grusovin, J., Stoichevska, V., Ryan, J. M., Castelli, L. A., and Ward, C. W. (2002). Cellular munc18c levels can modulate glucose transport rate and GLUT4 translocation in 3T3L1 cells. FEBS Lett. 528, 154-160. doi: 10.1016/S0014-5793(02)03279-9

Massart, J., Sjogren, R. J., Lundell, L. S., Mudry, J. M., Franck, N., O'Gorman, D. J., et al. (2017). Altered miRNA-29 expression in type 2 diabetes influences glucose and lipid metabolism in skeletal muscle. Diabetes 66, 1807-1818. doi: $10.2337 / \mathrm{db} 17-0141$

Mohamed, J. S., Hajira, A., Pardo, P. S., and Boriek, A. M. (2014). MicroRNA149 inhibits PARP-2 and promotes mitochondrial biogenesis via SIRT-1/PGC$1 \alpha$ network in skeletal muscle. Diabetes 63, 1546-1559. doi: $10.2337 / \mathrm{db} 13-$ 1364

Motulsky, H. J., and Brown, R. E. (2006). Detecting outliers when fitting data with nonlinear regression-a new method based on robust nonlinear regression and the false discovery rate. BMC Bioinformatics 7:123. doi: 10.1186/1471-2105-7-123

Nielsen, S., Scheele, C., Yfanti, C., Akerstrom, T., Nielsen, A. R., Pedersen, B. K., et al. (2010). Muscle specific microRNAs are regulated by endurance exercise in human skeletal muscle. J. Physiol. 588(Pt 20), 4029-4037. doi: 10.1113/jphysiol.2010.189860

Oh, Y. S., Cho, K. A., Ryu, S. J., Khil, L. Y., Jun, H. S., Yoon, J. W., et al. (2006). Regulation of insulin response in skeletal muscle cell by caveolin status. J. Cell. Biochem. 99, 747-758. doi: 10.1002/jcb.20943

Phielix, E., Meex, R., Moonen-Kornips, E., Hesselink, M. K., and Schrauwen, P. (2010). Exercise training increases mitochondrial content and ex vivo mitochondrial function similarly in patients with type 2 diabetes and in control individuals. Diabetologia 53, 1714-1721. doi: 10.1007/s00125-010-1764-2

Phielix, E., Meex, R., Ouwens, D. M., Sparks, L., Hoeks, J., Schaart, G., et al. (2012). High oxidative capacity due to chronic exercise training attenuates lipidinduced insulin resistance. Diabetes 61, 2472-2478. doi: 10.2337/db11-1832

Siri, W. E. (1956). The gross composition of the body. Adv. Biol. Med. Phys. 4, 239-280. doi: 10.1016/B978-1-4832-3110-5.50011-X

Smith, S. S., Kessler, C. B., Shenoy, V., Rosen, C. J., and Delany, A. M. (2013). IGFI $3^{\prime}$ untranslated region: strain-specific polymorphisms and motifs regulating IGF-I in osteoblasts. Endocrinology 154, 253-262. doi: 10.1210/en.2012-1476

Tseng, Y. H., Cypess, A. M., and Kahn, C. R. (2010). Cellular bioenergetics as a target for obesity therapy. Nat. Rev. Drug Discov. 9, 465-482. doi: $10.1038 / \mathrm{nrd} 3138$

van de Weijer, T., Phielix, E., Bilet, L., Williams, E. G., Ropelle, E. R., Bierwagen, A., et al. (2014). Evidence for a direct effect of the $\mathrm{NAD}^{+}$precursor Acipimox on muscle mitochondrial function in humans. Diabetes 64, 1193-1201. doi: $10.2337 / \mathrm{db} 14-0667$

Vosselman, M. J., Hoeks, J., Brans, B., Pallubinsky, H., Nascimento, E. B., van der Lans, A. A., et al. (2015). Low brown adipose tissue activity in endurancetrained compared with lean sedentary men. Int. J. Obes. (Lond) 39, 1696-1702. doi: 10.1038/ijo.2015.130

Yan, B., Zhu, C. D., Guo, J. T., Zhao, L. H., and Zhao, J. L. (2013). miR-206 regulates the growth of the teleost tilapia (Oreochromis niloticus) through the modulation of IGF-1 gene expression. J Exp. Biol. 216(Pt 7), 1265-1269. doi: 10.1242/jeb.079590

Zhang, Y., Zhao, Y. P., Gao, Y. F., Fan, Z. M., Liu, M. Y., Cai, X. Y., et al. (2015). Silencing miR-106b improves palmitic acid-induced mitochondrial dysfunction and insulin resistance in skeletal myocytes. Mol. Med. Rep. 11, 3834-3841. doi: 10.3892/mmr.2014.3124

Zhou, T., Meng, X., Che, H., Shen, N., Xiao, D., Song, X., et al. (2016). Regulation of insulin resistance by multiple MiRNAs via targeting the GLUT4 signalling pathway. Cell. Physiol. Biochem. 38, 2063-2078. doi: 10.1159/000445565

Conflict of Interest Statement: The authors declare that the research was conducted in the absence of any commercial or financial relationships that could be construed as a potential conflict of interest.

Copyright (C) 2017 Dahlmans, Houzelle, Jörgensen, Phielix, Lindeboom, Hesselink, Schrauwen and Hoeks. This is an open-access article distributed under the terms of the Creative Commons Attribution License (CC BY). The use, distribution or reproduction in other forums is permitted, provided the original author(s) or licensor are credited and that the original publication in this journal is cited, in accordance with accepted academic practice. No use, distribution or reproduction is permitted which does not comply with these terms. 